\title{
3D-printed personalised prostheses for bone defect repair and reconstruction following resection of metacarpal giant cell tumours
}

\author{
Lin Xu ${ }^{1,2 \#}$, Hao Qin ${ }^{2,3 \#}$, Zhilin Cheng ${ }^{2 \#}$, Wen-Bo Jiang ${ }^{4}$, Jia Tan $^{4}$, Xiang Luo ${ }^{2}$, Wenhua Huang ${ }^{1,5}$ \\ ${ }^{1}$ Department of Human Anatomy, School of Basic Medical Sciences, Guangxi Medical University, Nanning, China; ${ }^{2}$ Guangxi Clinical Research \\ Center for Digital Medicine and 3D Printing (Guigang City People's Hospital), Guigang, China; 'Department of Orthopedics, Gaozhou People's \\ Hospital, Maoming, China; ${ }^{4}$ Clinical and Translational Research Center for 3D Printing Technology, Shanghai Ninth People's Hospital, Shanghai \\ Jiao Tong University School of Medicine, Shanghai, China; ${ }^{5}$ National Key Discipline of Human Anatomy, Guangdong Provincial Key Laboratory \\ of Medical Biomechanics, Guangdong Engineering Research Center for Translation of Medical 3D Printing Application, School of Basic Medical \\ Sciences, Southern Medical University, Guangzhou, China \\ Contributions: (I) Conception and design: L Xu; (II) Administrative support: X Luo, W Huang; (III) Provision of study materials or patients: L Xu, H \\ Qin, Z Cheng, WB Jiang, J Tan; (IV) Collection and assembly of data: L Xu, H Qin, Z Cheng; (V) Data analysis and interpretation: L Xu, H Qin, Z \\ Cheng; (VI) Manuscript writing: All authors; (VII) Final approval of manuscript: All authors. \\ \#These authors contributed equally to this work. \\ Correspondence to: Wenhua Huang. Department of Human Anatomy, School of Basic Medical Sciences, Guangxi Medical University, 22 Shuangyong \\ Road Nanning, Nanning 530021, China. Email: huangwenhua2009@139.com; Xiang Luo. Guangxi Clinical Research Center for Digital Medicine \\ and 3D Printing (Guigang City People’s Hospital), Guigang 537100, China. Email: dr-luo@163.com.
}

Background: Conventional surgical treatment for metacarpal giant cell tumours (GCTs) includes lesion scraping followed by bone grafting or bone cement filling and en bloc resection followed by repair and reconstruction using a vascularised bone flap. However, these methods have inherent shortcomings, including a high postoperative recurrence rate and poor mechanical stability. 3D-printing techniques are increasingly being applied in medicine, and 3D-printed personalised prostheses have achieved good clinical effects in orthopaedic repair and reconstruction. We aimed to investigate the clinical effects of 3D-printed personalised prostheses for bone defect repair and reconstruction following resection of metacarpal GCTs.

Methods: Three patients with metacarpal GCTs were examined in a retrospective cohort study. Through preoperative planning, a 3D-printed personalised prosthesis was designed and created for bone defect repair and reconstruction after tumour resection. Prosthesis fit, limb function, pain on the affected side, and the occurrence of complications were evaluated postoperatively.

Results: Postoperative X-ray examination revealed a satisfactory fit of the $3 \mathrm{D}$-printed prosthesis in terms of bone defect size and overall metacarpal shape, as well as good transverse and longitudinal metacarpal arches. The patients also exhibited good function in the affected limb, with good flexion and extension functions in the carpal, metacarpophalangeal, and interphalangeal joints, plus the absence of obvious pain, tumour recurrence, and complications such as pathologic fractures and prosthetic loosening.

Conclusions: When using a 3D-printed personalised prosthesis for bone defect repair and reconstruction following resection of metacarpal GCTs, a good fit with the bone defect can be achieved during prosthetic installation when preoperative planning and design have been adequately performed. Therefore, threedimensionally printed personalised prostheses can serve as an effective method for the treatment of metacarpal GCTs.

Keywords: 3D printing; giant cell tumour (GCT); metacarpals; personalised prosthesis

Submitted Jul 09, 2021. Accepted for publication Aug 30, 2021.

doi: $10.21037 / \mathrm{atm}-21-3400$

View this article at: https://dx.doi.org/10.21037/atm-21-3400 


\section{Introduction}

Giant cell tumour (GCT) of the bone is a common benign but locally aggressive primary bone tumour. It is typically found in the distal femur, proximal tibia, proximal humerus, and distal radius, and less so in the spine, hands, and feet. GCTs of the hand account for $2-5 \%$ of all cases and often involve the metaphyses of the metacarpals $(1,2)$. Conventional surgical treatment for GCTs of the metacarpals includes lesion scraping followed by bone grafting, or bone cement filling and en bloc resection followed by repair and reconstruction using a vascularised bone flap. However, the inherent limitations of these methods include a high postoperative recurrence rate and poor mechanical stability, especially in patients with Campanacci grade III GCTs $(3,4)$. In recent years, en bloc resection followed by the use of a prosthesis for post-resection bone defect repair and reconstruction has become the preferred approach for GCT treatment among an increasing number of orthopedists. However, the poor fit of conventional prostheses leads to a high rate of complications such as implant loosening or failure, resulting in poor postoperative limb function among patients $(5,6)$. Furthermore, the low incidence of metacarpal GCTs suggests that conventionally configured prostheses are currently unavailable for this condition.

Medical applications of three-dimensional (3D) printing technologies are increasing, and 3D-printed prostheses have demonstrated high precision and personalisation and achieved good clinical effects in orthopaedic repair and reconstruction (7-9). However, research on the application of $3 \mathrm{D}$-printed personalised prostheses in bone defects after metacarpal GCT resection is relatively scarce, with only a handful of case studies reported in the literature (10). In the present study, we retrospectively evaluated three cases of metacarpal GCTs in which satisfactory clinical effects were obtained with bone defect repair using $3 \mathrm{D}$-printed personalised prostheses following tumour resection. We present the following article in accordance with the STROBE reporting checklist (available at https://dx.doi. org/10.21037/atm-21-3400).

\section{Methods}

\section{General data}

Data for three patients with metacarpal GCTs who underwent surgical treatment between May 2016 and December 2018 were retrospectively analysed (1 male,
2 females; age 26, 32, and 38 years, respectively). All patients had en bloc GCT resection followed by postresection bone repair and reconstruction using a $3 \mathrm{D}$-printed personalised prosthesis. All GCTs were classified as grade III according to the Campanacci grading system. The GCTs were located at the distal end of the third metacarpal bone in one patient and at the distal end of the fourth metacarpal bone in two patients (Table 1). Before surgery, all patients underwent radiographic examination, computed tomography (CT) scanning, magnetic resonance imaging (MRI), and preoperative biopsy. The pain status, range of motion (ROM), and grip strength of the affected hand were recorded. Follow-up was performed at 1, 3, 6, and 12 months postoperatively within the first year after surgery and once every 6 months thereafter.

This retrospective study was approved by the Ethical Committee of the Guigang City People's Hospital, Guigang, People's Republic of China, and was conducted according to the ethical standards of the Declaration of Helsinki (as revised in 2013). All patients and their family members received adequate information regarding the study and signed an informed consent form. Written informed consent was given by the patients to publish this paper.

\section{Preoperative planning and fabrication of $3 D$-printed personalised prostheses}

The prostheses were designed by research teams at the Clinical and Translational Research Center for 3D Printing Technology, Shanghai Ninth People's Hospital, Shanghai Jiao Tong University School of Medicine, and the Guangxi Clinical Research Center for Digital Medicine and 3D Printing, and were fabricated by Shanghai Shengshi Medical Devices Co., Ltd. First, the lesion site of each patient, including the phalanges and carpal joint of the affected hand, was scanned using 64-row spiral CT. The distance between the distal and proximal cortical bones at the tumour site was measured using CT data, and the distance between the distal and proximal ends of the tumour was measured using MRI data. By importing the CT data into Mimics software (version 17.0; Materialise NV, Belgium), a 3D model of the metacarpal that included the tumour was established to determine the line for osteotomy at approximately $0.3-0.5 \mathrm{~cm}$ from the proximal end of the tumour. Subsequently, the prosthesis was simulated and designed based on the actual defect and anatomy of the metacarpal. The designed prosthesis had a 3D smooth interface structure consisting of a head and a handle. A 
Table 1 Baseline characteristics of the three patients

\begin{tabular}{lccccccc}
\hline Patient no. & Age (years) & Sex & Affected side & $\begin{array}{c}\text { Campanacci } \\
\text { grade }\end{array}$ & Treatment & $\begin{array}{c}\text { Length of resected } \\
\text { bone (cm) }\end{array}$ & $\begin{array}{c}\text { Follow-up duration } \\
(\mathrm{months})\end{array}$ \\
\hline 1 & 32 & Female & Right & III & 3D-printed prosthesis & 2.45 & 24 \\
2 & 38 & Female & Left & III & 3D-printed prosthesis & 2.50 & 30 \\
3 & 26 & Male & Right & III & 3D-printed prosthesis & 2.50 & 36 \\
\hline
\end{tabular}

double-opening design was adopted for the head portion, with the openings facing upward for use as attachment points during the repair of the articular capsules of the metacarpophalangeal joint and the surrounding ligaments. The handle portion of the prosthesis was designed such that stability could be achieved by securing the handle to the remaining metacarpal bone using bone cement. Finally, the prosthesis was printed using titanium alloy powder (Ti6Al4V) with a 3D printer (Arcam A1; Arcam, Sweden) and was sterilised by autoclaving before use. The entire process, from surgical plan confirmation to prosthesis fabrication, was completed within 5 days.

\section{Surgical method}

The surgical procedure for each of the three patients was as follows. The patient was placed in the supine position and anaesthetised using brachial plexus blockade. A longitudinal incision, approximately $5 \mathrm{~cm}$ in length, was made along the dorsal side of the third or fourth metacarpal bone. After the extensor tendon had been retracted and the lesion had been fully exposed, osteotomy was performed along the preoperatively designed line for complete resection of the tumour lesion and partial resection of the articular capsules around the metacarpal. Rinsing was carried out three times with hydrogen peroxide, povidone-iodine, and saline, and tumour wall inactivation was performed using anhydrous alcohol. The proximal medullary cavity of the metacarpal was expanded by screw tapping and filled with bone cement to install the prosthesis. When the prosthesis was securely installed, the articular capsules of the metacarpophalangeal joint were repaired, a drainage tube was placed, and the incision was sutured layer-wise.

\section{Postoperative treatment and functional rebabilitation}

\section{Postoperative treatment}

After surgery, the operated wrist of each patient was placed in a cast in a neutral position, antibiotics were routinely administered intravenously for 2 days, and anti-inflammatory analgesics (celecoxib capsules) were orally administered for pain relief and swelling reduction. The surgery site was kept clean to prevent infection and prosthesis rejection.

\section{Functional rehabilitation}

From weeks 1 to 3 , all of the aforementioned postoperative treatments were continued. Progressive exercises were added as tolerated, considering the increase in oedema, pain, and stiffness after an exercise session. Blocking exercises were performed to improve independent tendon gliding. During this period, the patient was independent and took part in exercise, oedema, and pain management programmes. Upon wound closure, scar management was initiated as appropriate.

During week 4, gentle resistance activities of the long flexors and extensors were initiated. Static progressive splints were also used.

From weeks 6 to 12, progress exercises were maintained as tolerated. Splinting was discontinued when the joint ROM remained unchanged for 3 weeks and when passive ROM was easily maintained.

\section{Evaluation indicators}

The function of the affected limb was evaluated using the Disabilities of the Arm, Shoulder and Hand (DASH) questionnaire and the Musculoskeletal Tumor Society (MSTS) scoring system developed in 1993. Pain intensity was measured using a visual analogue scale (VAS) $(11,12)$, and the voluntary range of finger joint motion was evaluated using total active motion (TAM) (13). The postoperative pathologic findings of the tumour, occurrence of complications, and imaging evaluation results were recorded for each patient.

\section{Statistical method}

IBM SPSS Statistics for Windows version 22.0 (IBM Corp., 


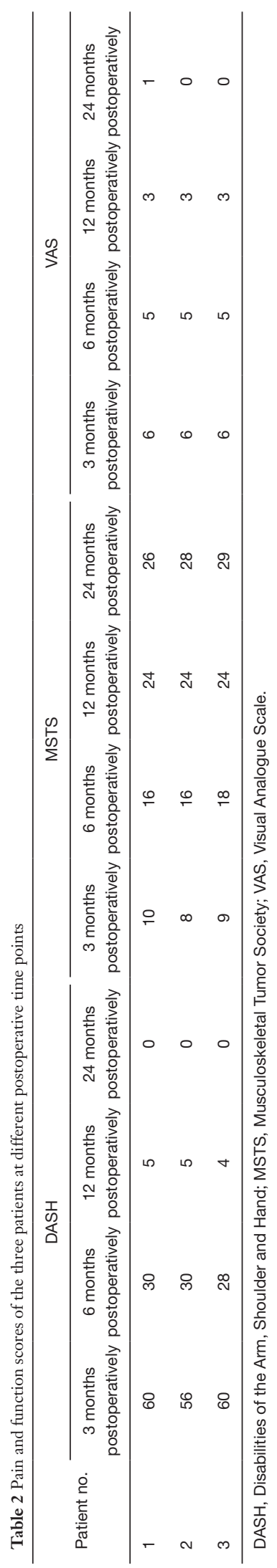

Armonk, NY, USA) was used for all frequency analysis.

\section{Results}

The surgical procedure proceeded smoothly in all three patients, with the incisions achieving stage I healing without early complications. Timely follow-up was performed postoperatively for all patients, with the final follow-up performed at 24, 30, and 36 months postoperatively. X-ray examination revealed a satisfactory fit of the $3 \mathrm{D}$-printed prostheses with respect to the bone defect size and overall metacarpal shape, and good transverse and longitudinal metacarpal arches. Table 2 shows the DASH, MSTS, and VAS scores of the patients at 3, 6, 12, and 24 months postoperatively. During the final follow-up, all patients exhibited good TAM in the affected limb, with good flexion and extension functions in the carpal, metacarpophalangeal, and interphalangeal joints (Table 3). All the patients achieved DASH scores of 0; their MSTS scores (26, 28, and 29) were excellent; and their VAS scores were 1, 0 , and 0 , which indicated the absence of evident pain in the affected limb. Tumour recurrence or complications such as pathologic fractures and prosthetic loosening were not observed.

\section{Typical case report}

A 32-year-old female had a GCT at the distal end of the right fourth metacarpal bone that invaded the fourth metacarpophalangeal joint over a disease duration of 6 months. Her clinical manifestations included swelling at the tumour site, evident pain upon local application of pressure, absence of pathologic fractures, limited flexion and extension of the right third to fifth metacarpophalangeal joints, local limitation of grip function, and decreased grip strength. A GCT diagnosis was considered based on the $\mathrm{X}$-ray, 3D-CT, and MRI examination results (Figure 1) and was confirmed by a biopsy (Figure 2).

The GCT was classified as grade III according to the Campanacci grading system. Three treatment options were initially considered. (I) Amputation: the patient and her family members were unwilling to accept amputation and expressed a strong desire for limb preservation. (II) Exclusion with bone cement followed by vascularised bone flap grafting plus metacarpophalangeal joint fusion: this procedure is highly complex and is associated with several complications. In addition, metacarpophalangeal joint fusion will result in a partial loss of function of the right hand and unpredictable long-term outcomes. (III) Massive 
Table 3 TAM of the three patients at 24 months postoperatively

\begin{tabular}{lcccc}
\hline & \multicolumn{3}{c}{ TAM $\left(^{\circ}\right)$} \\
\cline { 2 - 5 } Patient no. & $\begin{array}{c}\text { Carpal joint (extension and } \\
\text { flexion) }\end{array}$ & $\begin{array}{c}\text { MP joint (extension and } \\
\text { flexion) }\end{array}$ & $\begin{array}{c}\text { PIP joint (extension and } \\
\text { flexion) }\end{array}$ & $\begin{array}{c}\text { DIP joint (extension and } \\
\text { flexion) }\end{array}$ \\
\hline 1 & 150 & 90 & 90 & 80 \\
2 & 160 & 110 & 100 & 80 \\
3 & 160 & 110 & 100 & 80 \\
\hline
\end{tabular}

DIP, distal interphalangeal; MP, metacarpophalangeal; PIP, proximal interphalangeal; TAM, total active motion.
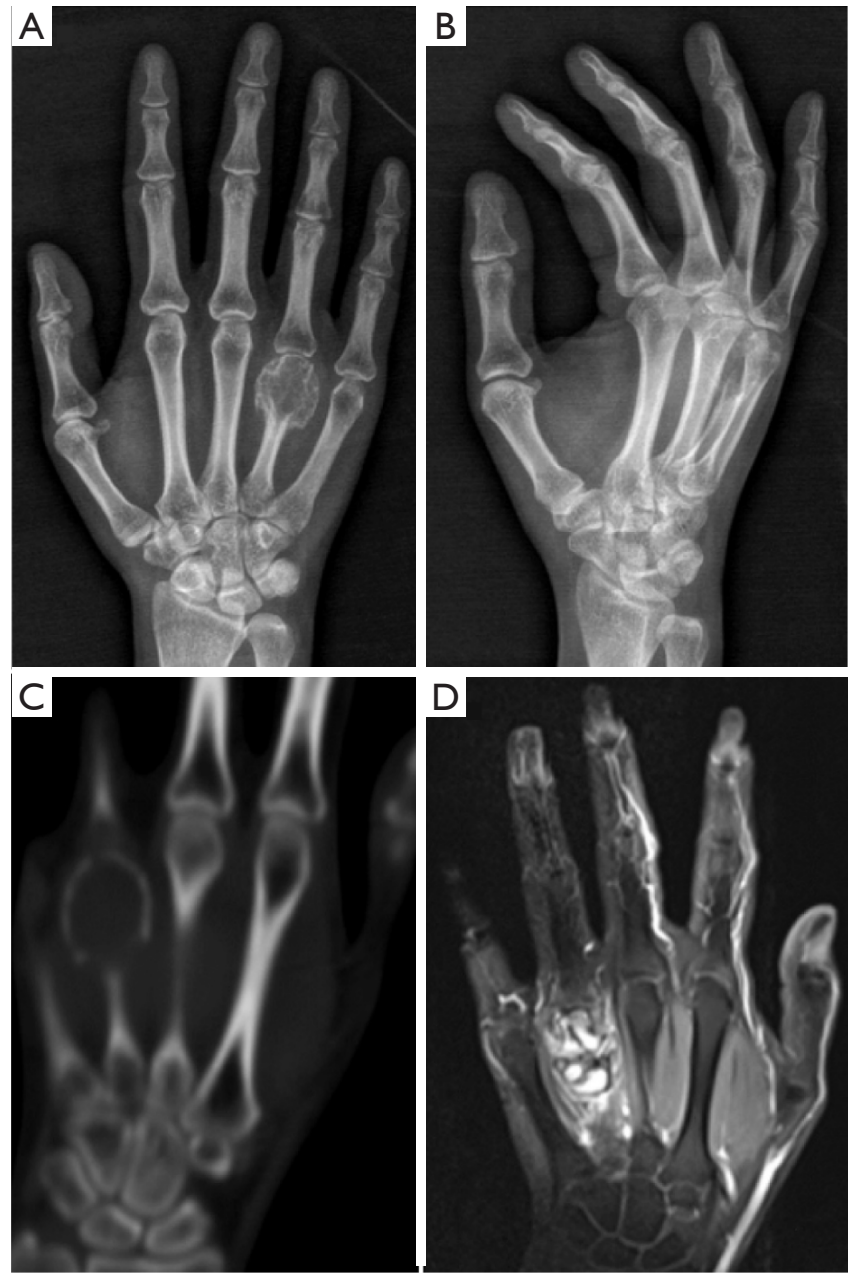

Figure 1 Preoperative X-ray, computed tomography (CT), and magnetic resonance imaging (MRI) of the right hand. (A) X-ray, Anterior-posterior (AP) view; (B) X-ray, Oblique (OB) view; (C) CT; (D) MRI.

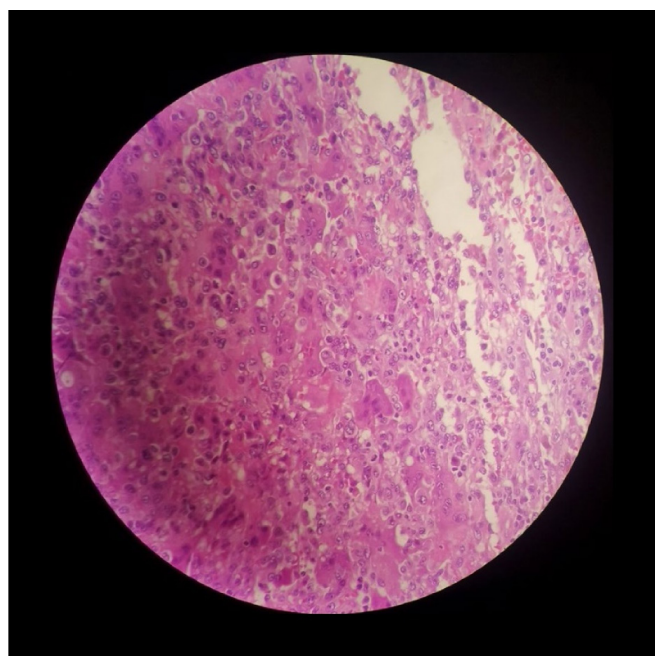

Figure 2 Microscopic view of the biopsy specimen (hematoxylin and eosin, $\times 400$ ).

bone allograft transplantation or allograft prosthesis transplantation: as allografts are susceptible to rejection and have poorer stability, these two methods lead to a high risk of bone resorption and fatigue fracture at a later stage. Hence, this surgical treatment option was ultimately rejected by the patient. After analysis of several treatment options, a joint decision to use a $3 \mathrm{D}$-printed personalised titanium alloy prosthesis for functional repair and reconstruction was made by the patient, her family members, and the doctor. With this method, the integrity of the affected hand could be preserved, a certain degree of motion could be maintained in the metacarpophalangeal joint, and the surgical procedure was simple and fast with few complications. Preoperative CT and MRI were performed for tumour area marking (Figure 3) and preoperative design of the osteotomy line at approximately $0.3-0.5 \mathrm{~cm}$ from the proximal end of 

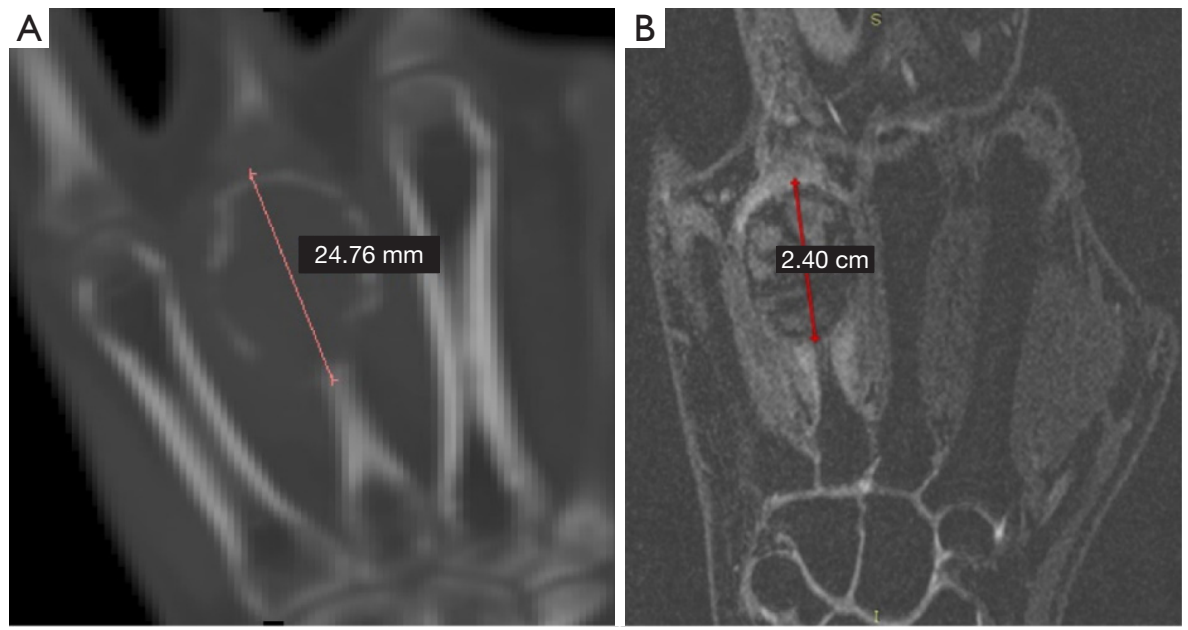

Figure 3 Preoperative tumour area marking on computed tomography (CT) and magnetic resonance imaging (MRI).

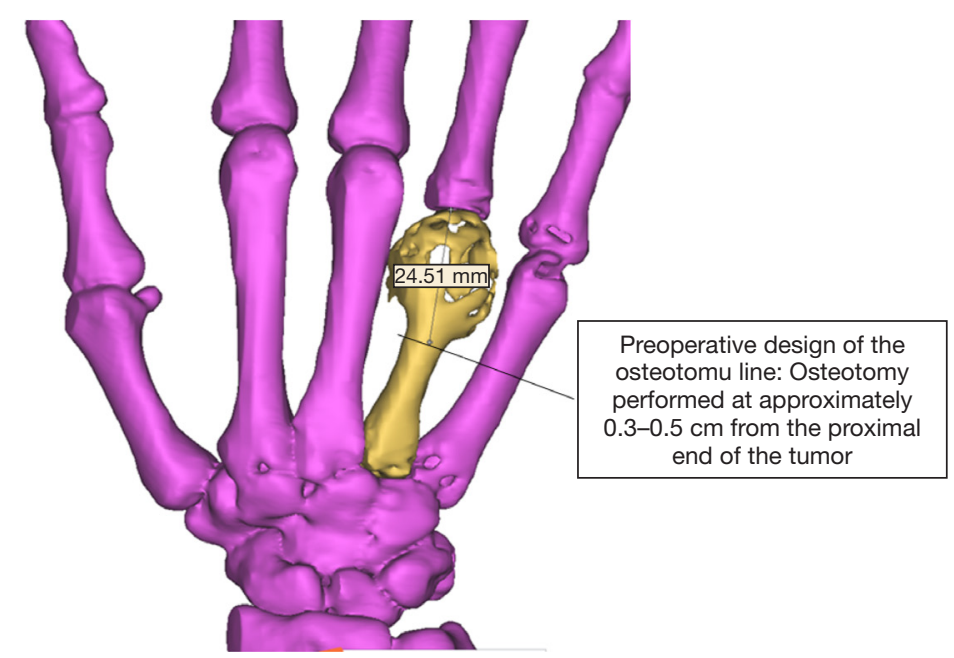

Figure 4 Preoperative design of the osteotomy line: approximately $0.3-0.5 \mathrm{~cm}$ from the proximal end of the tumour.

the tumour (Figure 4).

Figure 5 shows the prosthesis design and the 3D-printed titanium alloy prosthesis, and Figure 6 shows the intraoperative status and resected tumour.

Anteroposterior and lateral radiographs of both hands taken at 24 months postoperatively revealed a good overall fit between the prosthesis and the right metacarpal, and good transverse and longitudinal metacarpal arches (Figure 7).

During functional follow-up at 24 months postoperatively, the patient exhibited good function in the affected limb, with good flexion and extension functions in the carpal, metacarpophalangeal, and interphalangeal joints (Figure 8).

The DASH, MSTS, and VAS scores for the affected limb were 0,26 , and 1 , respectively. Postoperatively, a comprehensive set of functional rehabilitation measures was formulated for each patient based on their individual conditions to prevent postoperative joint stiffness that might result in poor limb function. At 24 months postoperatively, the patients exhibited good flexion and extension functions in the carpal, metacarpophalangeal, and interphalangeal joints. At 1-3 months postoperatively, the patients reported an evident dragging sensation at the prosthetic site and localised electric shock-like pain that was more pronounced at night or upon contact with the skin. During the early postoperative period, the skin on the affected hand exhibited elevated sensitivity to temperature 


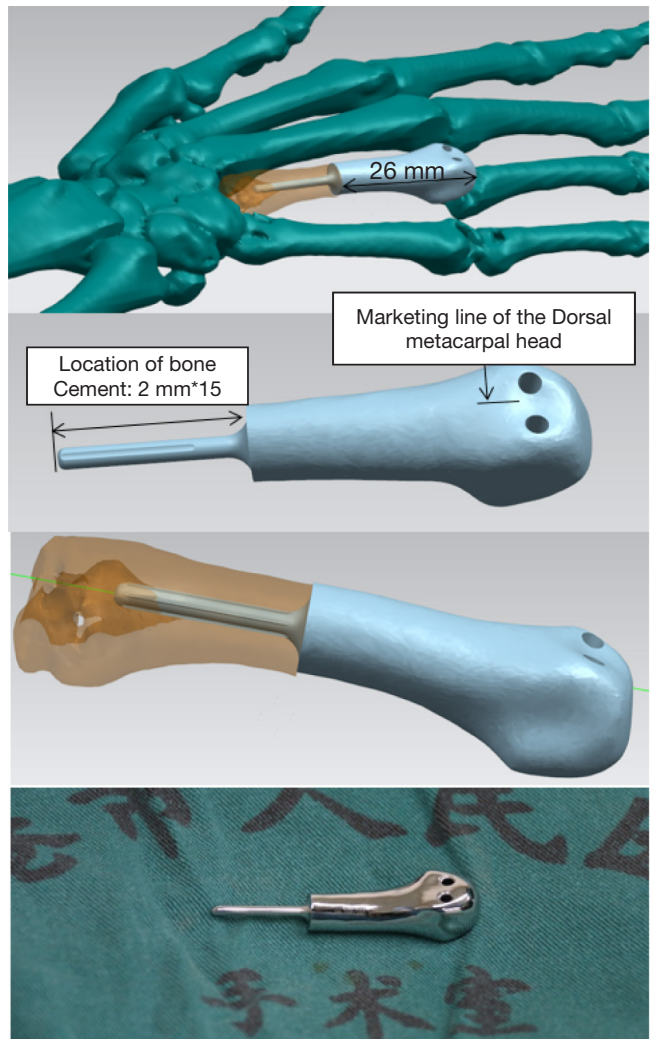

Figure 5 Prosthesis design and the $3 \mathrm{D}$-printed titanium alloy prosthesis. changes, with an evident tingling pain felt at temperatures $<15^{\circ} \mathrm{C}$ and pruritus at temperatures $>45^{\circ} \mathrm{C}$. However, these symptoms gradually decreased after 5 months, which might be related to intraoperative nerve traction. From 5 months postoperatively, patients were tasked with holding chopsticks and a pen and using a computer keyboard. During the early stage, patients experienced evident soreness and swelling sensation when holding chopsticks or a pen, with a marked increase in soreness, swelling, and fatigue felt in the hand after writing 200 Chinese characters. The patients were unable to perform key striking movements and experienced a dragging sensation in the palm when they first used the keyboard, but they became gradually accustomed after 2 weeks of persistent rehabilitation efforts. Patients reported a distinct sensation of tightness accompanied by crackling sounds in the metacarpophalangeal joints when performing gripping and stretching movements with the hand, resulting from postoperative joint adhesion or stiffness. These symptoms gradually disappeared from 6 months postoperatively onward. At 8 months postoperatively, strength training was initiated. Patients were able to lift objects weighing 5-7 kg by 12 months postoperatively, which was indicative of good limb function. All three patients obtained excellent postoperative MSTS scores and were satisfied with the overall treatment regimen (including
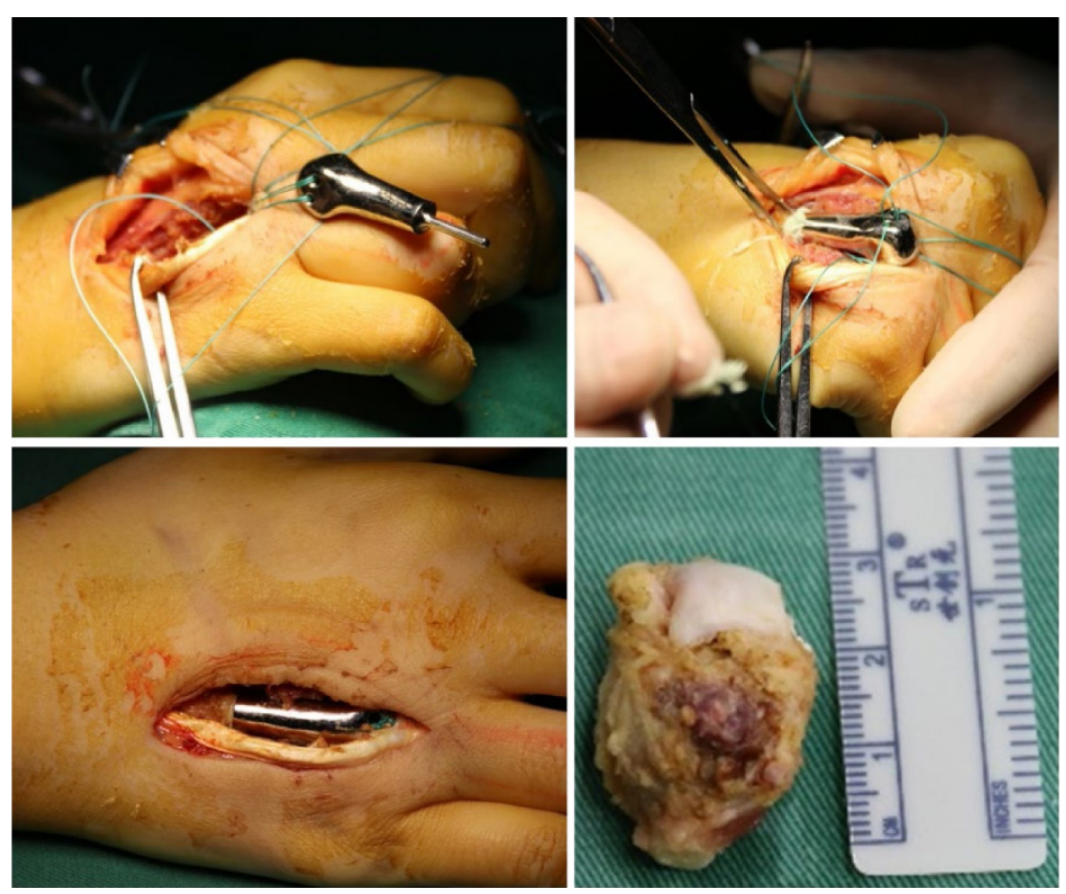

Figure 6 Intraoperative status and the resected tumour. 

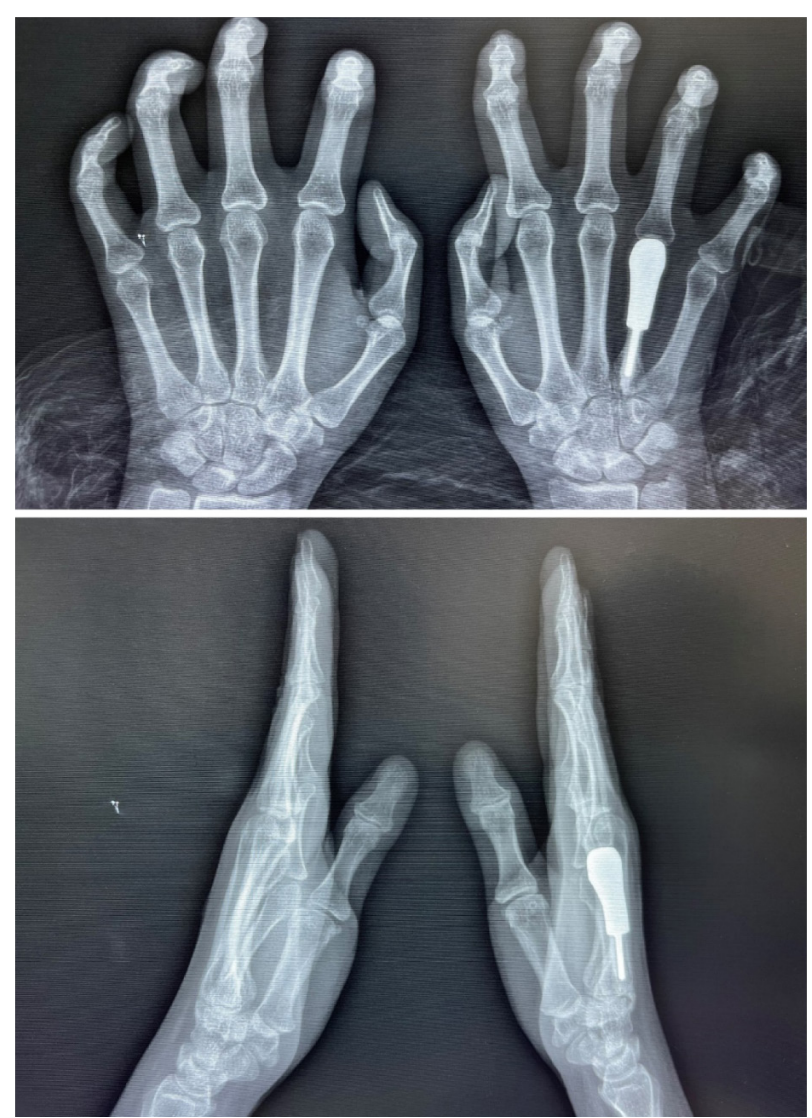

Figure 7 Anterior-posterior (AP) and lateral radiographs of both hands taken at 24 months postoperatively, showing good overall fit between the prosthesis and the right metacarpal, and good transverse and longitudinal metacarpal arches. the preoperative formulation of the surgical treatment plan and the postoperative functional rehabilitation programme). This indicates that our novel approach of using a $3 \mathrm{D}$-printed personalised metacarpal prosthesis for repair and reconstruction during GCT treatment may be more readily accepted by patients under most circumstances compared with traditional methods such as amputation. Nevertheless, a longer follow-up may be required for the validation of treatment effects.

\section{Discussion}

GCT of the bone is a common primary bone tumour with a predilection for the age group of $20-40$ years. It is typically found at the ends of long bones, especially in the distal femur and proximal tibia (2). The hand is a rare site of GCT occurrence, with the metacarpals and phalangeal epiphyses being involved in most cases, and reports of GCTs in the hand are relatively rare $(14,15)$. A PubMed search of the relevant literature yielded 23 published articles, which mainly consisted of case reports. A total of 35 metacarpal GCT cases have been reported, with the patients consisting of 23 males and 12 females in the age groups of $<20$ years (10 patients; $28.6 \%$ ), $20-40$ years (16 patients; $45.7 \%$ ), and $>40$ years ( 9 patients; $25.7 \%$ ). The left hand was affected in 17 patients, the right hand in 17 patients, and the side was unspecified in 1 patient. There were 34 patients with single tumours involving the first metacarpal (4 patients; $11.8 \%$ ), second metacarpal (5 patients; $14.7 \%$ ), third metacarpal

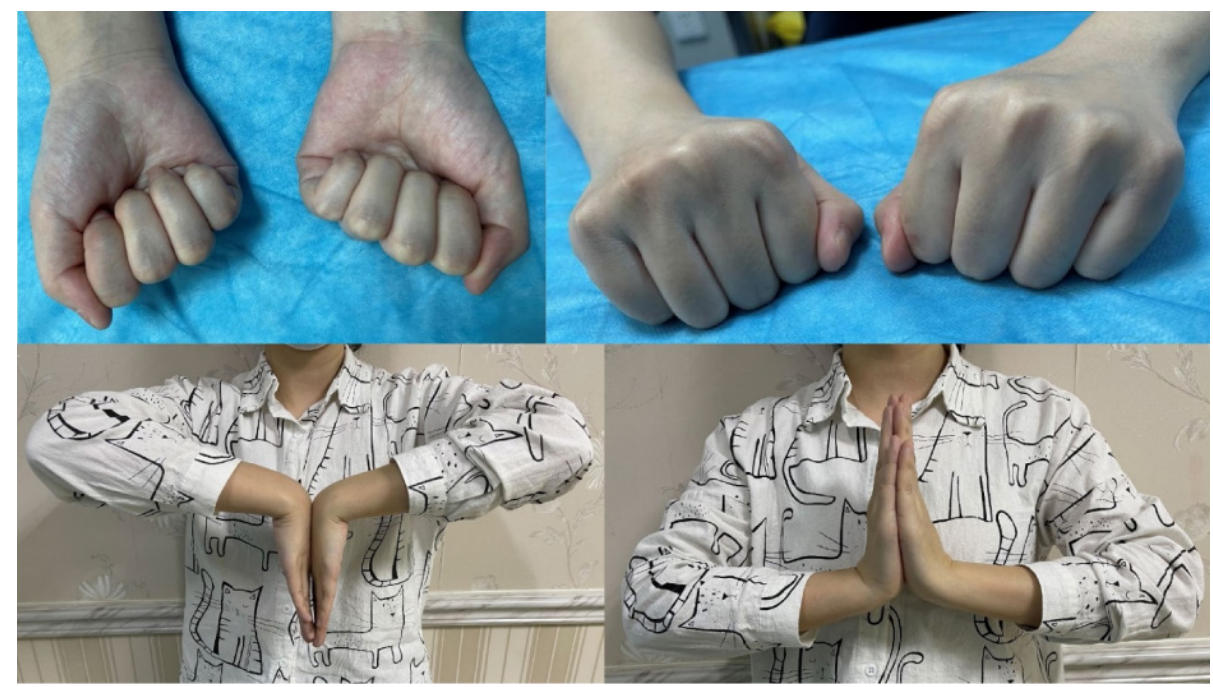

Figure 8 Functional follow-up at 24 months postoperatively. The patient exhibits good function in the affected limb, with good flexion and extension functions in the carpal, metacarpophalangeal, and interphalangeal joints. 
(6 patients; $17.6 \%$ ), fourth metacarpal (13 patients; $38.2 \%$ ), or fifth metacarpal (6 patients; $17.6 \%$ ), and 1 patient had multiple tumours involving the second, fourth, and fifth metacarpals. A review of the contents of the 23 articles revealed the several characteristics of metacarpal GCTs. (I) Most cases were single tumours with a predilection for the age group of $20-40$ years ( 46\%); however, patients aged $<20$ or $>40$ years also accounted for a significant number of the cases ( $29 \%$ and $\sim 26 \%$, respectively). (II) The common clinical manifestations included pain and swelling. The fourth metacarpal was most commonly involved ( 40\%), and the GCT incidence rates of the other metacarpals were similar among the remaining cases. Moreover, tumours invaded a large portion of the metacarpal in most cases. (III) At the time of diagnosis, the GCT in most patients was Campanacci grade II or III, in the progression stage, accompanied by evident bone destruction and extension to the metacarpal shaft and subchondral bone. (IV) Multifocal or multicentric manifestations were highly likely to occur. (V) Finally, compared with GCTs of long bones, metacarpal GCTs were more aggressive, exhibited a high recurrence rate, and were prone to metastatic spread to distant sites after multiple recurrences.

The conventional surgical treatment for metacarpal GCTs includes lesion scraping, followed by bone grafting or bone cement filling, and en bloc resection followed by repair and reconstruction using a vascularised bone flap. However, these methods have inherent limitations, including a high postoperative recurrence rate and poor mechanical stability (16). Scraping followed by bone grafting enables bone volume and joint preservation but is associated with a higher recurrence rate $(17,18)$. In recent years, en bloc resection followed by the use of a prosthesis for post-resection bone defect repair and reconstruction has become the preferred approach for GCT treatment among an increasing number of orthopedists. However, conventionally configured prostheses for metacarpal GCTs are currently unavailable due to the low incidence of such tumours, and studies on the use of prostheses for the repair of post-resection bone defects in patients with metacarpal GCTs have been limited to a handful of case reports. The application of $3 \mathrm{D}$-printing technologies in the field of orthopaedics has provided novel methods of bone defect repair and reconstruction following resection of metacarpal GCTs. This allows for wide or en bloc resection of metacarpal GCTs followed by the use of a preoperatively designed and fabricated 3D-printed personalised prosthesis for the repair and reconstruction of the post-resection bone defect. The perfect fit between the prosthesis and the defect site minimises the possibility of postoperative recurrence and facilitates maintenance of the hand's mechanical stability.

In the present study, 3D-printed personalised prostheses were used for bone defect repair and reconstruction after tumour resection in three patients with metacarpal GCTs. Postoperative X-ray examination results revealed a satisfactory fit of the 3D-printed prosthesis in terms of the bone defect size and overall metacarpal shape. During the final follow-up, the patients also exhibited good function in the affected limb, without evident pain, tumour recurrences, or complications such as pathologic fractures and prosthetic loosening. These results indicated that $3 \mathrm{D}$-printed personalised prostheses are an effective means of bone defect reconstruction after resection of metacarpal GCTs. As each $3 \mathrm{D}$-printed prosthesis was personalised based on actual patient conditions, a suitable fit with the postresection bone defect could be achieved for the satisfaction of anatomical and biomechanical requirements.

The key points for the preoperative design of $3 \mathrm{D}$-printed prostheses for bone defect repair and reconstruction after resection of metacarpal GCT are as follows. First, care was taken to attain a good shape and size match between the prosthesis and the post-resection bone defect. An excessively large prosthesis poses difficulties with implantation or requires the resection of a greater amount of normal bone tissue, which results in an enlarged bone defect, and increases the risk of postoperative pathologic fracture. If the designed prosthesis is too small, bone grafting will be required to achieve a good fit with the bone defect, which makes the prosthesis susceptible to loosening, hence failing to achieve the intended mechanical stabilisation. Second, our designed prosthesis had a 3D smooth interface structure and consisted of a head and a handle. A double-opening design was adopted for the head portion, with the openings facing upward for use as attachment points during the repair of the articular capsules of the metacarpophalangeal joint and the surrounding ligaments. The prosthesis was designed with a smooth surface to prevent or reduce friction between the prosthesis and the tendons, hence preventing tendon rupture.

This study has some limitations. First, it had a small sample size because of the low incidence of metacarpal GCTs. Therefore, further studies with a larger sample size are required to validate the benefits of our proposed treatment approach. Second, the follow-up duration was relatively short. Long-term follow-up of the patients of this 
study may be necessary to investigate recurrence and the survival outcomes associated with personalised prostheses. Finally, compared with bone grafting and bone cement, prosthesis fabrication by $3 \mathrm{D}$ printing and filling is more costly. In our future clinical work, we will gather more cases of metacarpal GCTs for further research.

\section{Conclusions}

The use of a 3D-printed personalised prosthesis for bone defect repair and reconstruction following resection of metacarpal GCTs, when combined with adequate preoperative planning and design to achieve a good fit between the installed prosthesis and the post-resection bone defect, is useful for the treatment of metacarpal GCT.

\section{Acknowledgments}

Funding: This work was supported by The Science and Technology Project of Guangdong Province [2016B090917001]; Guangxi Science and Technology Program [Guangxi Science No. AD,17195042, Guangxi Science No. AD,17129017]; Guigang Science and Technology Development Program [Guigang Science General No. 1834002]; and Seeds Fund of the Engineering Research Center of Digital Medicine of the Ministry of Education of Shanghai Jiao Tong University [No. 20210410].

\section{Footnote}

Reporting Checklist: The authors have completed the STROBE reporting checklist. Available at https://dx.doi. org/10.21037/atm-21-3400

Data Sharing Statement: Available at https://dx.doi. org/10.21037/atm-21-3400

Conflicts of Interest: All authors have completed the ICMJE uniform disclosure form (available at https://dx.doi. org/10.21037/atm-21-3400). The authors have no conflicts of interest to declare.

Ethical Statement: The authors are accountable for all aspects of the work in ensuring that questions related to the accuracy or integrity of any part of the work are appropriately investigated and resolved. This retrospective study was approved by the Ethical Committee of the Guigang City People's Hospital, Guigang, People's Republic of China (No. GYYXLL-201605), and was conducted according to the ethical standards of the Declaration of Helsinki of(as revised in 2013). All patients and their family members received adequate information regarding the study and signed an informed consent form. Written informed consent was given by the patients to publish this paper.

Open Access Statement: This is an Open Access article distributed in accordance with the Creative Commons Attribution-NonCommercial-NoDerivs 4.0 International License (CC BY-NC-ND 4.0), which permits the noncommercial replication and distribution of the article with the strict proviso that no changes or edits are made and the original work is properly cited (including links to both the formal publication through the relevant DOI and the license). See: https://creativecommons.org/licenses/by-nc-nd/4.0/.

\section{References}

1. von Borstel D, A Taguibao R, A Strle N, et al. Giant cell tumor of the bone: aggressive case initially treated with denosumab and intralesional surgery. Skeletal Radiol 2017;46:571-8.

2. Mohaidat ZM, Al-Jamal HZ, Bany-Khalaf AM, et al. Giant cell tumor of bone: Unusual features of a rare tumor. Rare Tumors 2019;11:2036361319878894.

3. Montgomery C, Couch C, Emory CL, et al. Giant Cell Tumor of Bone: Review of Current Literature, Evaluation, and Treatment Options. J Knee Surg 2019;32:331-6.

4. Zuo D, Zheng L, Sun W, et al. Contemporary adjuvant polymethyl methacrylate cementation optimally limits recurrence in primary giant cell tumor of bone patients compared to bone grafting: a systematic review and metaanalysis. World J Surg Oncol 2013;11:156.

5. Lv ZR, Li ZF, Yang ZP, et al. One-Step Reconstruction with a Novel Suspended, Modular, and 3D-Printed Total Sacral Implant Resection of Sacral Giant Cell Tumor with Preservation of Bilateral S1-3 Nerve Roots via a PosteriorOnly Approach. Orthop Surg 2020;12:58-66.

6. Wang Y, Min L, Lu M, et al. The functional outcomes and complications of different reconstruction methods for Giant cell tumor of the distal radius: comparison of Osteoarticular allograft and three-dimensional-printed prosthesis. BMC Musculoskelet Disord 2020;21:69.

7. Lu M, Min L, Xiao C, et al. Uncemented threedimensional-printed prosthetic replacement for giant cell tumor of distal radius: a new design of prosthesis and surgical techniques. Cancer Manag Res 2018;10:265-77. 
8. Damert HG, Altmann S, Kraus A. Custom-made wrist prosthesis in a patient with giant cell tumor of the distal radius. Arch Orthop Trauma Surg 2013;133:713-9.

9. Gan F, Luo X, Chen G, et al. Treatment of giant cell tumor of bone around knee joint with three-dimensional printing personalized prosthesis. Zhongguo Xiu Fu Chong Jian Wai Ke Za Zhi 2020;34:751-5.

10. Punyaratabandhu T, Lohwongwatana B, Puncreobutr C, et al. A Patient-Matched Entire First Metacarpal Prosthesis in Treatment of Giant Cell Tumor of Bone. Case Rep Orthop 2017;2017:4101346.

11. Sletten IN, Hellund JC, Olsen B, et al. Conservative treatment has comparable outcome with bouquet pinning of little finger metacarpal neck fractures: a multicentre randomized controlled study of 85 patients. J Hand Surg Eur Vol 2015;40:76-83.

12. Enneking WF, Dunham W, Gebhardt MC, et al. A system for the functional evaluation of reconstructive procedures after surgical treatment of tumors of the musculoskeletal

Cite this article as: $\mathrm{Xu} \mathrm{L}$, Qin H, Cheng Z, Jiang WB, Tan J, Luo X, Huang W. 3D-printed personalised prostheses for bone defect repair and reconstruction following resection of metacarpal giant cell tumours. Ann Transl Med 2021;9(18):1421. doi: 10.21037/atm-21-3400 system. Clin Orthop Relat Res 1993;(286):241-6.

13. Pan S, Gu Y, Shi D. Upper limb function evaluation of Hand Surgery Society of Chinese Medical Association. Chin J Hand Surg 2000;16:130-5.

14. Gachhayat AK, Patnaik S, Sahoo AK, et al. Giant Cell Tumor of Third Metacarpal: A Rare Case Report and Review of Literature. J Orthop Case Rep 2020;9:11-4.

15. Lewallen LW, Wagner ER, Moran SL. Giant Cell Tumor of the Metacarpal: Case Report. Hand (N Y) 2017;12:NP113-7.

16. Sailesh SS, Muthu S, Ismail NDM. Hemi-Fibular Grafting for Metacarpal Giant Cell Tumor - Surgical Technique. J Orthop Case Rep 2020;10:80-4.

17. Kotwal PP, Ansari MT, Mahmood A, et al. A management strategy for giant cell tumor of the metacarpal: A single-center series of 11 cases. J Clin Orthop Trauma 2020;11:657-61.

18. Saikia KC, Bhuyan SK, Ahmed F, et al. Giant cell tumor of the metacarpal bones. Indian J Orthop 2011;45:475-8. 